\title{
SYSTEME INTERNATIONAL
}

The following information on S.I. units and factors for conversion between S.I. and older conventional units is provided for the convenience of readers and authors. Reprints of these tables are available on request from The Canadian Anaesthetists' Socjety Journal, 178 St. George Street, Toronto, Canada, M5R 2M7.

BASIC SI UNITS

\begin{tabular}{lll}
\hline \hline Physical quantity & Name & Symbol \\
\hline Length & Metre & $\mathrm{m}$ \\
Mass & Kilogram & $\mathrm{kg}$ \\
Time & Second* & $\mathrm{s}$ \\
Electric current & Ampere & $\mathrm{A}$ \\
Thermodynamic temperature & Kelvin & $\mathrm{K}$ \\
Luminous intensity & Candela & $\mathrm{cd}$ \\
Amount of substance & Mole & mol \\
\hline
\end{tabular}

Minute (min), hour (h) and day (d) will remain in use although they are not official SI units.

Prefixes For SI UnITS

\begin{tabular}{llllll}
\hline Factor & Name & Symbol & Factor & Name & Symbol \\
\hline $10^{18}$ & Exa & $\mathrm{E}$ & $10^{-18}$ & Atto- & $\mathrm{a}$ \\
$10^{15}$ & Peta & $\mathrm{P}$ & $10^{-15}$ & Femto & $\mathrm{f}$ \\
$10^{12}$ & Tera- & $\mathrm{T}$ & $10^{-12}$ & Pico- & $\mathrm{p}$ \\
$10^{9}$ & Giga- & $\mathrm{G}$ & $10^{-9}$ & Nano- & $\mathrm{n}$ \\
$10^{6}$ & Mega- & $\mathrm{M}$ & $10^{-6}$ & Micro- & $\mathrm{H}$ \\
$10^{3}$ & Kilo- & $\mathrm{k}$ & $10^{-3}$ & Milli- & $\mathrm{m}$ \\
$10^{2}$ & Hecto- & $\mathrm{h}$ & $10^{-2}$ & Centi- & $\mathrm{c}$ \\
1 & Deca- & $\mathrm{da}$ & $10^{-1}$ & Deci- & $\mathrm{d}$ \\
\hline
\end{tabular}

Derived SI Units

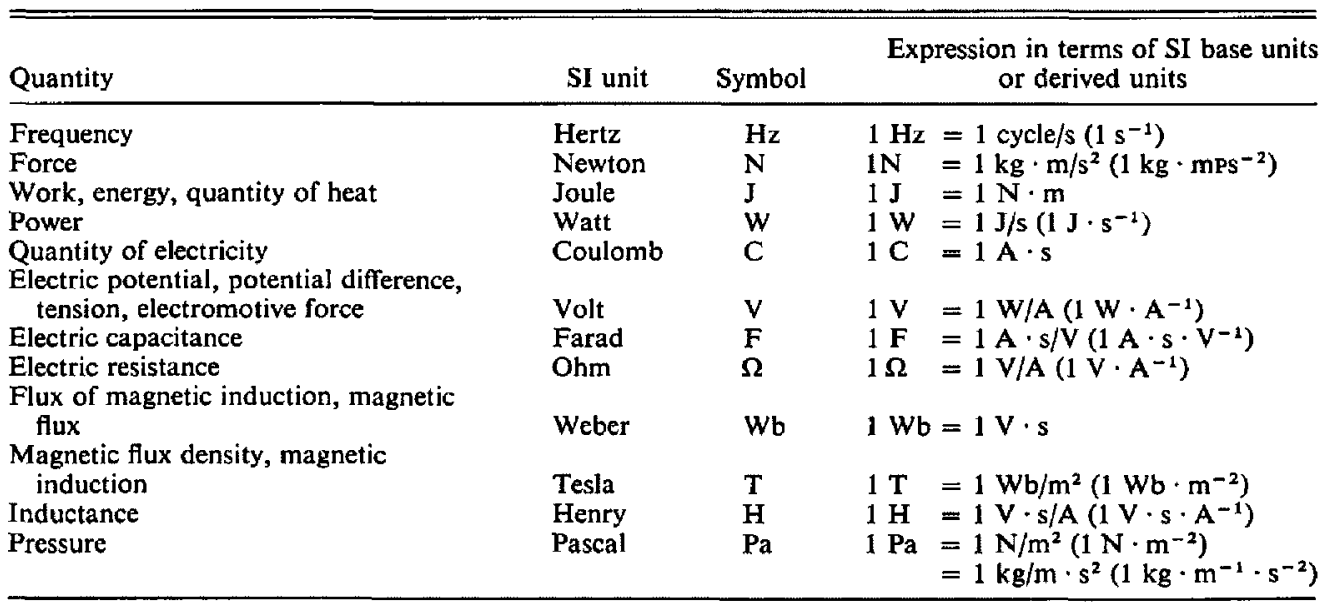

The litre $\left(10^{-3} \mathrm{~m}^{3}=\mathrm{dm}^{3}\right)$, though not official, will remain in use as a unit of volume as also will the dyne (dyn) as a unit of force (1 dyn $\left.=10^{-5} \mathrm{~N}\right)$. 


\begin{tabular}{|c|c|c|c|}
\hline \multirow[b]{2}{*}{ SI unit } & \multirow[b]{2}{*}{ Old Unit } & \multicolumn{2}{|c|}{ Conversion factors } \\
\hline & & $\begin{array}{l}\text { Old to SI } \\
\text { (exact) }\end{array}$ & $\begin{array}{l}\text { SI to old } \\
\text { (approx.) }\end{array}$ \\
\hline $\mathbf{k P a}$ & $\mathrm{mm} \mathbf{H g}^{*}$ & 0.133 & 7.5 \\
\hline $\mathbf{k P a}$ & $\begin{array}{l}1 \text { standard atmosphere } \dagger \\
\text { (approx: } 1 \text { Bar) }\end{array}$ & 101.3 & 0.01 \\
\hline $\mathrm{kPa}$ & $\mathrm{cmH}_{2} \mathrm{O}$ & 0.0981 & 10 \\
\hline $\mathbf{k P a}$ & $\mathrm{lbs} / \mathrm{sq}$ in & 0.145 & 7 \\
\hline
\end{tabular}

*e.g. systolic BP of $120 \mathrm{~mm} \mathrm{Hg}=16 \mathrm{kPa}$ and diastolic $\mathrm{BP}$ of $80 \mathrm{~mm} \mathrm{Hg}=$ $11 \mathrm{kPa}$.

$$
\dagger=760 \mathrm{~mm} \mathrm{Hg} \text {. }
$$

Blood Chemistry. Units and Conversion Factors

\begin{tabular}{|c|c|c|c|c|}
\hline \multirow[b]{2}{*}{ Measurement } & \multirow[b]{2}{*}{ SI unit } & \multirow[b]{2}{*}{ Old unit } & \multicolumn{2}{|c|}{ Conversion factors } \\
\hline & & & $\begin{array}{l}\text { Old to } S I \\
\text { (exact) }\end{array}$ & $\begin{array}{l}\text { SI to old } \\
\text { (approx.) }\end{array}$ \\
\hline \multicolumn{5}{|l|}{$\begin{array}{l}\text { Blood } \\
\text { Acid-Base }\end{array}$} \\
\hline $\mathrm{PCO}_{2}$ & $\mathbf{k P a}$ & $\mathrm{mm} \mathrm{Hg}$ & 0.133 & 7.5 \\
\hline & $\mathrm{kPa}$ & $\mathrm{mm} \mathrm{Hg}$ & 0.133 & 7.5 \\
\hline \multirow{2}{*}{\multicolumn{5}{|c|}{ Standard }} \\
\hline & $\mathrm{mmol} /$ litre & $\mathrm{mEq} /$ litre & \multirow{2}{*}{\multicolumn{2}{|c|}{$\begin{array}{l}\text { Numerically equivalent } \\
\text { Numerically equivalent }\end{array}$}} \\
\hline Base excess & $\mathrm{mmol} /$ litre & $\mathrm{mEq} /$ litre & & \\
\hline Glucose & $\mathrm{mmol} /$ litre & $\mathrm{mg} / 100 \mathrm{ml}$ & 0.0555 & 18 \\
\hline \multicolumn{5}{|l|}{ Plasma } \\
\hline Sodium & $\mathrm{mmol} /$ litre & $\mathrm{mEq} /$ litre & \multirow{2}{*}{\multicolumn{2}{|c|}{$\begin{array}{l}\text { Numerically equivalent } \\
\text { Numerically equivalent }\end{array}$}} \\
\hline \multirow{4}{*}{$\begin{array}{l}\text { Potassium } \\
\text { Magnesium } \\
\text { Chloride } \\
\text { Phosphate } \\
\text { (inorganic) }\end{array}$} & $\mathrm{mmol} /$ litre & $\mathrm{mEq} /$ litre & & \\
\hline & $\mathrm{mmol} /$ litre & $\mathrm{mEq} /$ litre & \multirow{2}{*}{\multicolumn{2}{|c|}{$\begin{array}{lc}0.5 & 2 \\
\text { Numerically equivalent }\end{array}$}} \\
\hline & $\mathrm{mmol} /$ litre & $\mathrm{mEq} /$ litre & & \\
\hline & mmolliter & & & \\
\hline Creatinine & mmol/litre & $\begin{array}{l}\mathrm{mEq} / \mathrm{ltre} \\
\mathrm{mg} / 100 \mathrm{ml}\end{array}$ & $\begin{array}{l}0.523 \\
88.4\end{array}$ & $\begin{array}{l}5.0 \\
0.01\end{array}$ \\
\hline Urea & $\mathrm{mmol} / \mathrm{litre}$ & $\mathrm{mg} / 100 \mathrm{ml}$ & 0.166 & 6.0 \\
\hline \multicolumn{5}{|l|}{ Serum } \\
\hline Calcium & $\mathrm{mmol} /$ litre & $\mathrm{mg} / 100 \mathrm{ml}$ & 0.25 & 4.0 \\
\hline Iron & $\mu \mathrm{mol} /$ litre & $\mu \mathrm{g} / 100 \mathrm{~mol}$ & 0.179 & 5.6 \\
\hline Bilirubin & $\mu \mathrm{mol} / \mathrm{litre}$ & $\mathrm{mg} / 100 \mathrm{ml}$ & 17.1 & 0.06 \\
\hline Cholesterol & $\mathrm{mmol} / \mathrm{litre}$ & $\mathrm{mg} / 100 \mathrm{ml}$ & 0.0259 & 39 \\
\hline Total proteins & g/litre & $\mathrm{g} / 100 \mathrm{ml}$ & 10.0 & 0.1 \\
\hline Albumin & $\mathbf{g}$ /litre & $\mathrm{g} / 100 \mathrm{ml}$ & 10.0 & 0.1 \\
\hline Globulin & $\mathrm{g} /$ litre & $\mathrm{g} / 100 \mathrm{mI}$ & 10.0 & 0.1 \\
\hline
\end{tabular}

Biochemical Content of Other Body Fluids

\begin{tabular}{|c|c|c|c|c|}
\hline \multirow[b]{2}{*}{ Measurement } & \multirow[b]{2}{*}{ SI unit } & \multirow[b]{2}{*}{ Old unit } & \multicolumn{2}{|c|}{ Conversion factors } \\
\hline & & & $\begin{array}{l}\text { Old to SI } \\
\text { (exact) }\end{array}$ & $\begin{array}{l}\text { SI to old } \\
\text { (approx.) }\end{array}$ \\
\hline $\begin{array}{l}\text { Urine } \\
\text { Calcium } \\
\text { Creatinine } \\
\text { Potassium } \\
\text { Sodium }\end{array}$ & $\begin{array}{l}\mathrm{mmol} / 24 \mathrm{~h} \\
\mathrm{mmol} / 24 \mathrm{~h} \\
\mathrm{mmol} / \mathrm{litre} \\
\mathrm{mmol} / \text { litre }\end{array}$ & $\begin{array}{l}\mathrm{mg} / 24 \mathrm{~h} \\
\mathrm{mg} / 24 \mathrm{~h} \\
\mathrm{mEq} / \text { litre } \\
\mathrm{mEq} / \mathrm{litre}\end{array}$ & $\begin{array}{l}0.025 \\
0.00884 \\
\text { Numeric } \\
\text { Numeric }\end{array}$ & $\begin{array}{r}40 \\
113 \\
\text { alent } \\
\text { alent }\end{array}$ \\
\hline $\begin{array}{l}\text { Cerebro-spinal fluid } \\
\text { Protein } \\
\text { Glucose }\end{array}$ & $\begin{array}{l}\mathrm{g} / \text { litre } \\
\mathrm{mmol} / \text { litre }\end{array}$ & $\begin{array}{l}\mathrm{mg} / 100 \mathrm{ml} \\
\mathrm{mg} / 100 \mathrm{ml}\end{array}$ & $\begin{array}{l}0.01 \\
0.0555\end{array}$ & $\begin{array}{r}100 \\
18\end{array}$ \\
\hline
\end{tabular}


Haematology

\begin{tabular}{|c|c|c|c|c|}
\hline \multirow[b]{2}{*}{ Measurement } & \multirow[b]{2}{*}{ SI units } & \multirow[b]{2}{*}{ Old unit } & \multicolumn{2}{|c|}{ Conversion factors } \\
\hline & & & Old to SI & SI to old \\
\hline Haemoglobin $(\mathrm{Hb})$ & $\mathrm{g} / \mathrm{dl}$ & $\mathrm{g} / 100 \mathrm{ml}$ & \multicolumn{2}{|c|}{ Numerically equivalent } \\
\hline Packed cell volume & No unit* & Per cent & 0.01 & 100 \\
\hline Mean cell Hb conc. & $\mathrm{g} / \mathrm{dl}$ & Per cent & \multicolumn{2}{|c|}{ Numerically equivalent } \\
\hline Mean cell $\mathrm{Hb}$ & $\mathrm{pg}$ & $\mu \mu \mathrm{g}$ & \multicolumn{2}{|c|}{ Numerically equivalent } \\
\hline Red cell count & Cells/litre & Cells $/ \mathrm{mm}^{3}$ & $10^{6}$ & $10^{-6}$ \\
\hline White cell count & Cells litre & Cells $/ \mathrm{mm}^{3}$ & $10^{6}$ & $10^{-6}$ \\
\hline Reticulocytes & Per cent & Per cent & \multicolumn{2}{|c|}{ Numerically equivalent } \\
\hline Platelets & Cells/litre & Cells $/ \mathrm{mm}^{3}$ & $10^{6}$ & $10^{-6}$ \\
\hline
\end{tabular}

*Expressed as decimal fraction, e.g. normal adult male value 0.40 to 0.54 .

\begin{tabular}{cc}
\multicolumn{2}{c}{$\begin{array}{c}\text { PH AND NMOL/LITRE OF } \\
\mathbf{H}^{+} \text {ACTIVITY }\end{array}$} \\
\hline \hline $\mathrm{pH}$ & nmol/litre \\
\hline 6.80 & 158 \\
6.90 & 126 \\
7.00 & 100 \\
7.10 & 79 \\
7.20 & 63 \\
7.25 & 56 \\
7.30 & 50 \\
7.35 & 45 \\
7.40 & 40 \\
7.45 & 36 \\
7.50 & 32 \\
7.55 & 28 \\
7.60 & 25 \\
7.70 & 20 \\
\hline
\end{tabular}




\section{SYSTEME INTERNATIONAL}

Pour le bénéfice des auteurs et des lecteurs, nous publions ci-dessous, les tables de conversion des unités de mesures anciennes en Système International, et autres renseignements pertinents. On peut obtenir des reproductions de ces tables de conversion sur simple demande écrite adressée au Canadian Anaesthetists' Society Journal, 178 St. George Street, Toronto, Canada, M5R 2M7.

UNITÉS SI DE BASE

\begin{tabular}{lll}
\hline \hline \multicolumn{1}{c}{ Quantité physique } & Nom & Symbole \\
\hline Longueur & Mètre & $\mathrm{m}$ \\
Masse & Kilogramme & $\mathrm{Kg}$ \\
Temps & Seconde* & $\mathrm{s}$ \\
Courant électrique & Ampère & $\mathrm{A}$ \\
Température thermodynamique & Kelvin & $\mathrm{K}$ \\
Intensité lumineuse & Candela & $\mathrm{cd}$ \\
Quantité de substance & Mole & $\mathrm{mol}$ \\
\hline
\end{tabular}

*Les unités manute $(\mathrm{mn})$, heure $(\mathrm{h})$ et jour ( $\mathrm{j})$ continuent d'être employées sans être des unités SI.

Préfixes Utilisés en SI

\begin{tabular}{llcllc}
\hline \hline Facteur & Nom & Symbole & Facteur & Nom & Symbole \\
\hline $10^{18}$ & Exa & $\mathrm{E}$ & $10^{-18}$ & Atto- & $\mathrm{a}$ \\
$10^{15}$ & Peta & $\mathrm{P}$ & $10^{-15}$ & Femto & $\mathrm{f}$ \\
$10^{12}$ & Tera- & $\mathrm{T}$ & $10^{-12}$ & Pico- & $\mathrm{p}$ \\
$10^{9}$ & Giga- & $\mathrm{G}$ & $10^{-9}$ & Nano- & $\mathrm{n}$ \\
$10^{6}$ & Mega- & $\mathrm{M}$ & $10^{-6}$ & Micro- & $\mathrm{\mu}$ \\
$10^{3}$ & Kilo- & $\mathrm{k}$ & $10^{-3}$ & Milli- & $\mathrm{m}$ \\
$10^{2}$ & Hecto- & $\mathrm{h}$ & $10^{-2}$ & Centi- & $\mathrm{c}$ \\
$\mathrm{l}$ & Deca- & $\mathrm{da}$ & $10^{-1}$ & Deci- & $\mathrm{d}$ \\
\hline
\end{tabular}

UNITÉS SI DÉRIVÉS

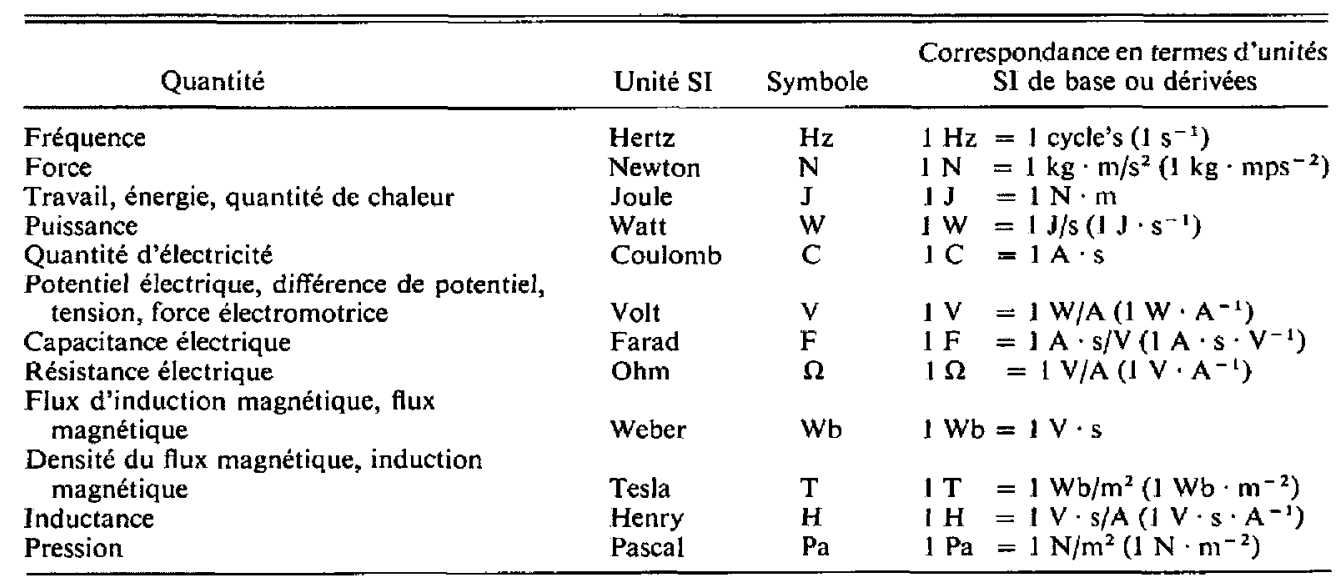

Le litre $\left(10^{-3} \mathrm{~m}^{3}=\mathrm{dm}^{3}\right)$, sans officiellement appartenir au Système International, continue de s'employer comme unité de volume comme la dyne $\left(\right.$ dyn) comme unité de force $\left(1 \mathrm{dyn}=10^{-5} \mathrm{~N}\right)$. 


\begin{tabular}{|c|c|c|c|}
\hline \multirow[b]{2}{*}{ Unité SI } & \multirow[b]{2}{*}{ Unité ancienne } & \multicolumn{2}{|c|}{ Facteurs de conversion } \\
\hline & & $\begin{array}{c}\text { Ancienne à SI } \\
\text { (exact) }\end{array}$ & $\begin{array}{c}\text { SI à ancienne } \\
\text { (approx.) }\end{array}$ \\
\hline $\mathrm{kPa}$ & $\mathrm{mm} \mathbf{H g}^{*}$ & 0,133 & 7,5 \\
\hline $\mathrm{kPa}$ & $\begin{array}{l}1 \text { atmosphère }(760 \mathrm{~mm} \mathrm{Hg}) \\
\text { (approx.: I Bar) }\end{array}$ & 101,3 & 0,01 \\
\hline $\mathrm{kPa}$ & $\mathrm{cmH}_{2} \mathrm{O}$ & 0,0981 & 10 \\
\hline $\mathrm{kPa}$ & $\mathrm{lbs} / \mathrm{po}^{2}$ & 0,145 & 7 \\
\hline
\end{tabular}

*v.g. pression artérielle systolique de $120 \mathrm{~mm} \mathrm{Hg}=16 \mathrm{kPa}$ et la pression diastolique de $80 \mathrm{~mm} \mathrm{Hg}=11 \mathrm{kPa}$.

Chimie SANGuine. Unités et facteurs de CONVERSION

\begin{tabular}{|c|c|c|c|c|}
\hline \multirow[b]{2}{*}{ Mesure } & \multirow[b]{2}{*}{ Unité SI } & \multirow[b]{2}{*}{ Unité ancienne } & \multicolumn{2}{|c|}{ Facteurs de conversion } \\
\hline & & & $\begin{array}{l}\text { Ancienne à SI } \\
\text { (exact) }\end{array}$ & $\begin{array}{l}\text { SI à ancienne } \\
\text { (approx.) }\end{array}$ \\
\hline \multicolumn{5}{|l|}{$\begin{array}{l}\text { Sang } \\
\text { Equilibre } \\
\quad \text { acide-base }\end{array}$} \\
\hline $\mathrm{PCO}_{2}$ & $\mathrm{kPa}$ & $\mathrm{mm} \mathrm{Hg}$ & 0,133 & 7,5 \\
\hline $\mathrm{PO}_{2}$ & $\mathrm{kPa}$ & $\mathrm{mm} \mathrm{Hg}$ & 0,133 & 7,5 \\
\hline Bicarbonate standard & $\mathrm{mmol} /$ litre & $\mathrm{mEq} /$ litre & \multirow{2}{*}{\multicolumn{2}{|c|}{$\begin{array}{l}\text { Numériquement équivalent } \\
\text { Numériquement équivalent }\end{array}$}} \\
\hline Excès de base & $\mathrm{mmol} /$ litre & $\mathrm{mEq} /$ litre & & \\
\hline Glucose & $\mathrm{mmol} /$ litre & $\mathrm{mg} / 100 \mathrm{ml}$ & 0,0555 & 18 \\
\hline \multicolumn{5}{|l|}{ Plasma } \\
\hline Sodium & $\mathrm{mmol} /$ litre & $\mathrm{mEq} / \mathrm{litre}$ & \multirow{2}{*}{\multicolumn{2}{|c|}{$\begin{array}{l}\text { Numériquement équivalent } \\
\text { Numériquement équivalent }\end{array}$}} \\
\hline Potassium & $\mathrm{mmol} /$ litre & $\mathrm{mEq} / \mathrm{litre}$ & & \\
\hline Magnésium & $\mathrm{mmol} /$ litre & $\mathrm{mEq} / \mathrm{litre}$ & \multirow{2}{*}{\multicolumn{2}{|c|}{$\begin{array}{l}0,5 \\
\text { Numériquement équivalent }\end{array}$}} \\
\hline Chlorure & $\mathrm{mmol} /$ litre & $\mathrm{mEq} /$ litre & & \\
\hline \multicolumn{5}{|l|}{$\begin{array}{l}\text { Phosphate } \\
\text { (ingrganioue) }\end{array}$} \\
\hline (inorganique) & $\mathrm{mmol} /$ /itre & $\mathrm{mEq} / \mathrm{litre}$ & 0,323 & 3,0 \\
\hline Créatinine & $\mu \mathrm{mol} /$ litre & $\mathrm{mg} / 100 \mathrm{ml}$ & 88,4 & 0,01 \\
\hline Urée & $\mathrm{mmol} / \mathrm{litre}$ & $\mathrm{mg} / 100 \mathrm{ml}$ & 0,166 & 6,0 \\
\hline \multicolumn{5}{|l|}{ Sérum } \\
\hline Calcium & $\mathrm{mmol} /$ litre & $\mathrm{mg} / 100 \mathrm{ml}$ & 0,25 & 4,0 \\
\hline Fer & $\mu \mathrm{mol} / \mathrm{litre}$ & $\mu \mathrm{g} / 100 \mathrm{~mol}$ & 0,179 & 5,6 \\
\hline Bilirubine & $\mu \mathrm{mol} /$ litre & $\mathrm{mg} / 100 \mathrm{ml}$ & 17,1 & 0,06 \\
\hline Cholestérol & $\mathrm{mmol} /$ litre & $\mathrm{mg} / 100 \mathrm{ml}$ & 0,0259 & 39 \\
\hline Protéines totales & g/litre & $\mathrm{g} / 100 \mathrm{ml}$ & 10,0 & 0,1 \\
\hline Albumine & g/litre & $\mathrm{g} / 100 \mathrm{ml}$ & 10,0 & 0,1 \\
\hline Globuline & g/litre & $\mathrm{g} / 100 \mathrm{ml}$ & 10,0 & 0,1 \\
\hline
\end{tabular}

MESURE BIOCHIMIQUE DANS D'AUTRES LIQUIDES ORGANIQUES

\begin{tabular}{|c|c|c|c|c|}
\hline \multirow[b]{2}{*}{ Mesure } & \multirow[b]{2}{*}{ Unité SI } & \multirow[b]{2}{*}{ Unité ancienne } & \multicolumn{2}{|c|}{ Facteurs de conversion } \\
\hline & & & $\begin{array}{c}\text { Ancienne à SI } \\
\text { (exact) }\end{array}$ & $\begin{array}{c}\text { SI à ancienne } \\
\text { (approx.) }\end{array}$ \\
\hline \multicolumn{5}{|l|}{ Urine } \\
\hline Calcium & $\mathrm{mmol} / 24 \mathrm{~h}$ & $\mathrm{mg} / 24 \mathrm{~h}$ & 0,025 & 40 \\
\hline Créatinine & $\mathrm{mmol} / 24 \mathrm{~h}$ & $\mathrm{mg} / 24 \mathrm{~h}$ & 0,00884 & 113 \\
\hline Potassium & $\mathrm{mmol} /$ litre & $\mathrm{mEq} /$ litre & \multirow{2}{*}{\multicolumn{2}{|c|}{$\begin{array}{l}\text { Numériquement équivalent } \\
\text { Numériquement équivalent }\end{array}$}} \\
\hline Sodium & $\mathrm{mmol} /$ litre & $\mathrm{mEq} /$ litre & & \\
\hline \multicolumn{5}{|l|}{$\begin{array}{l}\text { Liquide } \\
\quad \text { céphalo-rachidien }\end{array}$} \\
\hline Protéine & g/litre & $\mathrm{mg} / 100 \mathrm{ml}$ & 0,01 & 100 \\
\hline Glucose & $\mathrm{mmol} / \mathrm{litre}$ & $\mathrm{mg} / 100 \mathrm{ml}$ & 0,0555 & 18 \\
\hline
\end{tabular}


Hématologie

\begin{tabular}{|c|c|c|c|c|}
\hline \multirow[b]{2}{*}{ Mesure } & \multirow[b]{2}{*}{ Unité SI } & \multirow[b]{2}{*}{ Unité ancienne } & \multicolumn{2}{|c|}{ Facteurs de conversion } \\
\hline & & & $\begin{array}{l}\text { Ancienne à SI } \\
\text { (exact) }\end{array}$ & $\begin{array}{l}\text { SI à ancienne } \\
\text { (approx.) }\end{array}$ \\
\hline Hémoglobine $(\mathrm{Hb})$ & $\mathrm{g} / \mathrm{dl}$ & $\mathrm{g} / 100 \mathrm{ml}$ & \multicolumn{2}{|c|}{ Numériquement équivalent } \\
\hline $\begin{array}{l}\text { Volume du culot } \\
\text { globulaire } \\
\text { Hb cellulaire moyenne }\end{array}$ & Pas d'inité* & Pour cent & 0,01 & 100 \\
\hline (concentrée) & $\mathrm{g} / \mathrm{dl}$ & Pour cent & \multirow{2}{*}{\multicolumn{2}{|c|}{$\begin{array}{l}\text { Numériquement équivalent } \\
\text { Numériquement équivalent }\end{array}$}} \\
\hline Hb cellulaire moyenne & & $\mu \mu \mathrm{g}$ & & \\
\hline Décompte globules rouges & Cellules/litre & Cellules $/ \mathrm{mm}^{3}$ & $10^{6}$ & $10^{-6}$ \\
\hline Décompte globules blancs & Cellules/litre & Cellules $/ \mathrm{mm}^{3}$ & $10^{6}$ & $10^{-6}$ \\
\hline Réticulocytes & Pour cent & Pour cent & \multicolumn{2}{|c|}{ Numériquement équivalent } \\
\hline Plaquettes & Cellules/litre & Cellules $/ \mathrm{nm}^{3}$ & $10^{6}$ & $10^{-6}$ \\
\hline
\end{tabular}

*S'exprime en fraction décimale, v.g. valeur normale d'un homme adulte 0,40 à 0,54 .

$\begin{gathered}\text { TABLE DE CONVERSION DE } \\
\text { pH EN NMOL/LITRE } \\
\text { D'ACTIVITÉ ION H }\end{gathered}$
\begin{tabular}{cc} 
DH & nmol/litre \\
\hline \hline pH & 158 \\
6,80 & 126 \\
6,90 & 100 \\
7,00 & 79 \\
7,10 & 63 \\
7,20 & 56 \\
7,25 & 50 \\
7,30 & 45 \\
7,35 & 40 \\
7,40 & 36 \\
7,45 & 32 \\
7,50 & 28 \\
7,55 & 25 \\
7,60 & 20 \\
7,70 & \\
\hline
\end{tabular}

